\title{
PROBABILITY AND QUASI-MEASURES - A NEW INTERPRETATION
}

\author{
JOHAN F. AARNES and ALF B. RUSTAD
}

\section{Introduction}

Let $X$ be a compact Hausdorff space, and let $\mathscr{C}$ (respectively $\mathcal{O}$ ) denote the collection of closed (respectively open) subsets of $X$. Let $\mathscr{A}=\mathscr{C} \cup \mathcal{O}$. A quasi-measure in $X$ is a function $\mu: \mathscr{A} \rightarrow \mathrm{R}^{+}$which is monotone, additive and regular. More precisely we have:

(i) $\mu\left(A_{1}\right) \leq \mu\left(A_{2}\right)$ if $A_{1} \subset A_{2}$

(ii) $\mu\left(\biguplus_{i=1}^{n} A_{i}\right)=\sum_{i=1}^{n} \mu A_{i}(\biguplus$ indicates disjoint union, and we assume all $A_{i}$ and $\biguplus_{i=1}^{n} A_{i}$ in $\left.\mathscr{A}\right)$

(iii) $\mu U=\sup \{\mu C: C \subset U ; C \in \mathscr{C}\}$ for all $U$ in $\mathcal{O}$.

One may show that a quasi-measure has a (necessarily unique) extension to a regular Borel measure in $X$ if and only if it is subadditive on $\mathscr{C}$, i.e. if it satisfies

(iv) $\mu\left(C_{1} \cup C_{2}\right) \leq \mu C_{1}+\mu C_{2}$ for all $C_{1}, C_{2}$ in $\mathscr{C}$.

The whole point here is that quasi-measures that do not satisfy condition (iv) exist. Their basic construction has been given in [1], [2] and [3]. The main construction result ([1], Theorem 5.1) assumes that a set function $\mu$ initially is given on a fundamental family of sets $\mathscr{A}_{s}$, called the solid sets, and extended to all of $\mathscr{A}$ (a set $A \in \mathscr{A}$ is solid if $A$ and its compliment are both connected). A function $\mu: \mathscr{A}_{s} \rightarrow \mathbf{R}^{+}$is a solid set-function if it satisfies

(A) $\sum_{i=1}^{n} \mu C_{i} \leq \mu C$ whenever $\biguplus_{i=1}^{n} C_{i} \subset C$; $C_{i}, C \in \mathscr{C}_{s}$ for $i=1,2, \ldots, n$

(B) $\mu U=\sup \left\{\mu C: C \subset U ; C \in \mathscr{C}_{s}\right\}$ for all $U \in \mathcal{O}_{s}$

(C) $\mu A+\mu(X \backslash A)=\mu X$

Here $\mathscr{C}_{s}$ (respectively $\mathcal{O}_{s}$ ) denotes the family of closed (respectively open) solid sets in $X$. We assume here that $X$ is connected and locally path connected, and for simplicity we will also require that it has genus $g=0$ (for details see [1]). These conditions will be met in standard spaces like balls and spheres, for instance. The main construction theorem now states that each

Received April 11, 1997. 
solid set function has a unique extension to a quasi-measure in $X$. By this result, the construction problem is reduced to that of obtaining the solid setfunctions. It is the purpose of this note to describe how to do this by means of applying certain functions to existing (Borel or quasi-) measures. By this process we also obtain a new interpretation in probability theory, presented in the final section of this paper.

\section{Functions composed with measures}

We first introduce the class of functions we are going to consider.

Definition 2.1. A function $f:[0,1] \rightarrow[0,1]$ is called a $q$-function if it is continuous from the right and satisfies

1. $f(0)=0, f(x-)+f(1-x)=1$

2. $\sum_{i=1}^{n} f\left(x_{i}\right) \leq f\left(\sum_{i=1}^{n} x_{i}\right)$ whenever $x_{1}, x_{2}, \ldots, x_{n} \in[0,1]$ and $\sum_{i=1}^{n} x_{i}<1$.

Let $\nu$ be a normalized Borel (or quasi-) measure in $X$, i.e. $\nu(X)=1$. We say that $\nu$ is on-splitting if there is no disjoint pair $C_{1}, C_{2} \in \mathscr{C}_{s}$ such that $\nu C_{1}>0, \nu C_{2}>0$ and $\nu C_{1}+\nu C_{2}=1$. For instance, Lebesgue-measure on the unit disk, or the unit sphere (normalized) is non-splitting.

Proposition 2.2. Let $f$ be a q-function, and let $\nu$ be a normalized regular Borel (or quasi-) measure in X. Define $\mu$ on $\mathscr{A}_{s}$ by: $\mu C=f(\nu C) ; C \in \mathscr{C}_{s}$ and $\mu U=1-\mu(X \backslash U) ; U \in \mathcal{O}_{s}$. If either $\nu$ is non-splitting or $f$ is continuous, then $\mu$ is a solid set-function.

Proof. We first verify that $f$ is non-decreasing: Let $0 \leq x<y<1$. By 2.1.2: $f(x) \leq f(x)+f(y-x) \leq f(x+(y-x))=f(y)$. Also, by 2.1.1: $f(1-)+$ $f(0)=1 \Rightarrow f(1-)=1$. Putting $x=0$ in 2.1.1 yields $f(0)+f(1)=1 \Rightarrow$ $f(1)=1$. Then we verify condition (A) for a solid set-function: Let $C_{1}, \ldots, C_{n}, C \in \mathscr{C}_{s}$ and suppose $\biguplus_{i=1}^{n} C_{i} \subset C$. First if $\sum_{i=1}^{n} \nu C_{i}<1$ then by 2.1.2

$$
\sum \mu C_{i}=\sum f\left(\nu C_{i}\right) \leq f\left(\sum \nu C_{i}\right)=f\left(\nu\left(\biguplus C_{i}\right)\right) \leq f(\nu C)=\mu C
$$

since $f$ is non-decreasing. Now if $\sum \nu C_{i}=1$ and $\nu$ is non-splitting then $n=1$. This follows from lemma 3.3 in [1]. So, if $\sum \nu C_{i}=1$ we may assume that $f$ is continuous. Condition 2.1.1 then yields $f(x)+f(1-x)=1$ for all $x \in[0,1]$ so that $\sum \mu C_{i} \leq 1=\mu C$. Next, to show that $\mu$ is regular (condition (B)) we first note that if $C \subset U ; C \in \mathscr{C}_{s}, U \in \mathcal{O}_{s}$, then $\mu C \leq \mu U$. For if $C^{\prime}=X \backslash U$ then $C \cap C^{\prime}=\emptyset$ and it follows from the argument above that $\mu C+\mu C^{\prime} \leq 1 \Rightarrow \mu C \leq 1-\mu C^{\prime}=\mu U$. Now let $U \in \mathcal{O}_{s}$ be arbitrary. Since $\nu$ is (inner) regular we get 


$$
\begin{aligned}
\mu U & =1-\mu(X \backslash U)=1-f(\nu(X \backslash U)) \\
& =1-f(1-\nu U)=f(\nu U-) \\
& =\sup _{C \subset U} f(\nu C)=\sup _{C \subset U} \mu C
\end{aligned}
$$

The last equality follows from Lemma 3.1 and 3.1 in [2], which implies that for each compact $C \subset U \in \mathcal{O}_{s}$ there is a solid compact set $C^{\prime} \subset U$ with $C \subset C^{\prime}$. We have now verified conditions $(A)$ and $(B)$ for a solid set-function. Finally, condition $(C)$ holds trivially by construction.

We next turn to the question of determining which functions that are $q$ functions.

Lemma 2.3. Let $f:[0,1] \rightarrow[0,1]$ be a function satisfying:

1. $f(0)=0, f(x)+f(1-x)=1$

2. $\frac{f(x)}{x}$ is increasing on $\left(0, \frac{1}{2}\right]$

Then $f$ is non-decreasing and satisfies

(*) $\quad \sum_{i=1}^{n} f\left(x_{i}\right) \leq f\left(\sum_{i=1}^{n} x_{i}\right)$ whenever $\sum_{i=1}^{n} x_{i} \leq 1, x_{1}, \ldots, x_{n} \in[0,1] ; n \in \mathbf{Z}^{+}$.

Proof. Let $0<c<\frac{1}{2}$ and let $l=l(x)$ be the straight line through the origin and the point $(c, f(c))$. By condition 2.2 we have

$$
\begin{aligned}
& f(x) \leq l(x) \text { if } 0 \leq x \leq c \\
& f(x) \geq l(x) \text { if } c \leq x \leq \frac{1}{2}
\end{aligned}
$$

We first prove $(*)$ when $n=2$.

i) Let $0<x_{1} \leq x_{2} ; x_{1}+x_{2} \leq \frac{1}{2}$. Taking $c=x_{2}$ we get $f\left(x_{1}\right)+f\left(x_{2}\right) \leq$ $l\left(x_{1}\right)+l\left(x_{2}\right)=l\left(x_{1}+x_{2}\right) \leq f\left(x_{1}+x_{2}\right)$.

ii) $0<x_{1} \leq x_{2} \leq \frac{1}{2} ; \quad x_{1}+x_{2} \geq \frac{1}{2}$. By 2.3 .1 we get $f(x) \leq x$ if $x \leq \frac{1}{2}$ and $f(x) \geq x$ if $x \geq \frac{1}{2}$. Hence $f\left(x_{1}\right)+f\left(x_{2}\right) \leq x_{1}+x_{2} \leq f\left(x_{1}+x_{2}\right)$.

iii) $0<x_{1}<\frac{1}{2}<x_{2}<1 ; x_{1}+x_{2} \leq 1$. We have $0 \leq 1-x_{1}-x_{2} \leq \frac{1}{2}$, and $\left(1-x_{1}-x_{2}\right)+x_{1}=1-x_{2}<\frac{1}{2}$, so by 2.3 .1 and case i) above we get

$$
\begin{aligned}
f\left(1-x_{1}-x_{2}\right)+f\left(x_{1}\right) & \leq f\left(1-x_{2}\right), \text { i.e. } \\
1-f\left(x_{2}+x_{1}\right)+f\left(x_{1}\right) & \leq 1-f\left(x_{2}\right) \\
\Rightarrow f\left(x_{1}\right)+f\left(x_{2}\right) & \leq f\left(x_{1}+x_{2}\right)
\end{aligned}
$$

This establishes $(*)$ when $n=2$. An easy induction argument shows that it is true in general.

Corollary 2.4. Let $f:[0,1] \rightarrow[0,1]$ be continuous, convex on $\left[0, \frac{1}{2}\right]$ and 
satisfy $f(0)=0 ; f(x)+f(1-x)=1$. Then $\mu A=f(\nu A) ; A \in \mathscr{A}_{s}$ is a solid setfunction for each normalized quasi-measure $\nu$ in $X$.

Proof. The proof follows from lemma 2.

REMARK 1. As described in the introduction, each solid set-function extends to a unique quasi-measure in $X$. In general the quasi-measures obtained by q-functions will be proper quasi-measures, they are not subadditive. To illustrate this, let $X=D=$ unit disk, and let $\nu$ be normalized Lebesgue measure. If $f$ is a continuous, convex q-function which is not the identity function, we must have $f\left(\frac{1}{4}\right)<\frac{1}{4}$. Let $C_{1}$ and $C_{2}$ be disks in $X$ with area $\nu\left(C_{1}\right)=\nu\left(C_{2}\right)=\frac{1}{4}$. Assume $C_{1} \cap C_{2} \neq \emptyset$ and $C_{1} \cup C_{2} \in \mathscr{C}_{s}$. We have $\mu C_{1}+\mu C_{2}=f\left(\nu C_{1}\right)+f\left(\nu C_{2}\right)=2 f\left(\frac{1}{4}\right)<\frac{1}{2}$. On the other hand, by making $\nu\left(C_{1} \cap C_{2}\right)$ small we can have $\nu\left(C_{1} \cup C_{1}\right) \rightarrow \frac{1}{2}$, and then by the continuity of $f$ we can get $\mu\left(C_{1} \cup C_{2}\right)=f\left(\nu\left(C_{1} \cup C_{2}\right)\right)>\mu C_{1}+\mu C_{2}$. Hence $\mu$ is not subadditive.

We conclude this section with some examples.

EXAMPLE 2.5. Let $n \in \mathbf{Z}^{+}$be arbitrary, and let

$$
\begin{aligned}
I_{k} & =\left[\frac{k}{n+1}, \frac{k+1}{n+1}\right) ; k=0,1, \ldots, n-1 \\
I_{n} & =\left[\frac{n}{n+1}, 1\right]
\end{aligned}
$$

Define $f(x)=\frac{k}{n}$ on $I_{k} ; k=0, \ldots, n$. Then $f$ is a $q$-function. If $\nu$ is a non-splitting regular Borel measure we therefore obtain a quasi-measure $\mu$. If $n=1 \mu$ is simple, i.e. it only takes the values 0 and 1 . For general $n$ one may show that $\mu$ is an extreme point in the set $Q(X)$ of all normalized quasi-measures in $X$.

EXAMPLE 2.6. $f(x)=\sin ^{2}\left(\frac{\pi}{2} x\right)=\frac{1}{2}(1-\cos \pi x)$ is a continuous $q$-function which is convex on $\left[0, \frac{1}{2}\right]$.

ExAmple 2.7. $p(x)=3 x^{2}-2 x^{3}$ and $q(x)=2 x^{2}+2 x^{3}-5 x^{4}+2 x^{5}$ are polynomials of the type above.

\section{Quasi-measures and probability}

A normalized quasi-measure in $X$ is called a quasi-probability. The preceding section has shown how one may construct quasi-probabilities from $q$-functions and a given probability measure $\nu$ in $X$. We formalize this procedure. Let $Q(X)$ denote the set of quasi-probabilities in $X$ and let $q[0,1]$ denote the set of $q$-functions. Both these sets are convex. If $\nu$ is a fixed non-splitting 
element of $Q(X)$ we obtain a map $F_{\nu}: q[0,1] \rightarrow Q(X)$ by defining $\mu=F_{\nu}(f)$ where $\mu$ is given by Proposition 2.2 on $\mathscr{A}_{s}$ and then extended to all of $\mathscr{A}$ (Theorem 5.1 in [1]). Since $\mu$ is uniquely determined by its values on $\mathscr{A}_{s}$ it follows that $F_{\nu}$ is an affine map so the range of $F_{\nu}$ is a convex subset of $Q(X)$ which we will denote by $Q_{\nu}(X)$, and consists of the quasi-probabilities that are associated with $\nu$. Note that if $\nu$ is simple then $Q_{\nu}(X)$ only consists of $\nu$, i.e. $F_{\nu}(f)=\nu$ for all $f \in q[0,1]$. Our goal here is to give a probabilistic interpretation of the quasi-probabilities that are associated with an ordinary probability $\nu$. This will be done by considering concrete examples. A more general approach to quasi-probabilities may be found in [4].

EXAmPle 3.1. (Quasi-probability) Let $D$ be the unit disk, and let $\nu$ be the normalized Lebesgue measure in $D$. Then $\left(D^{3}, \mathscr{B}\left(D^{3}\right), \nu^{3}\right)$ where $\mathscr{B}\left(D^{3}\right)$ is the Borel sets in $D^{3}$ is a probability space. Further let $X_{i}$ be the $i^{\prime}$ th projection map for $i=1,2,3$ (i.e. $\left.X_{i}\left(x_{1}, x_{2}, x_{3}\right)=x_{i}\right)$. Then $\left\{X_{i}\right\}$ are independent random variables on $D^{3}$. Hence $P\left(\bigcap_{i=1}^{3} X_{i} \in A_{i}\right)=P\left(X_{1} \in A_{1}\right) \cdot P\left(X_{2} \in A_{2}\right)$. $P\left(X_{3} \in A_{3}\right)$ for any triple $\left\{A_{i}\right\}_{i=1}^{3} \subset \mathscr{B}(D)$, where of course $P\left(X_{i} \in A_{i}\right)=$ $\nu^{3}\left(X_{i}^{-1}\left(A_{i}\right)\right)=\nu\left(A_{i}\right) ; i=1,2,3$. We define a set function $\mu: \mathscr{A}_{s} \rightarrow[0,1]$ on the solid subsets of $D$ by

$$
\mu(A)=P\left(X_{i} \in A \text { for at least two values of } i\right)
$$

$\mu$ may be calculated combinatorially considering $D^{3}$ with the $X_{i}$ occurring respectively in the three disks. We then obtain $\mu(A)=\nu(A)^{3}+$ $\left(\frac{3}{2}\right) \nu(A)^{2} \nu(D \backslash A)=3 \nu(A)^{2}-2 \nu(A)^{3}$. Notice that $\mu \in Q_{\nu}(X)$ by the $q$-function $p(x)$ in Example 1 and hence determines a unique quasi-measure in $D$. One might be tempted to think that $\mu$ determines a new probability measure on $D$ but this is not so (see Remark 1). It is not difficult to imagine a situation where the set-function $\mu$ is interesting: Imagine an airdrop of three objects where in order for the drop to be successful you need to find two objects and the ground you cover searching is a solid set.

REMARK 2. Although the specific problem above can be solved with ordinary probability theory one should bear in mind that this is a very simple example to illustrate that the quasi-measures arises naturally in probability theory. Note that the construction above could analogously be done on the sphere. The resulting quasi-measure $\mu$ would then be rotation invariant and yet still not a measure. The "quasi" behavior of $\mu$ appears when the sets get larger.

EXAMPLE 3.2. In the example above the observations are made in triples where we can split the triple into three independent variables. However experimental statisticians often face the problem of choosing a model for dependent observations. In the example above this can be illustrated by the 
three points being charged particles dropped simultaneously onto the disk. The standard approach is then to try to determine how the observations are dependent, an approach often without success. Again consider observations on the disk in triples. After a series of experiments one might find an estimate for $\mu$ in (1). For instance, $F_{\nu}(f)$ where $\nu$ is the Lebesgue measure and $f$ is the function in Example 2.6 could be a suitable model. A statistical interpretation of this model can be that the three points are less likely to cluster in small disks than if they were independent. This can be seen from the positivity of the function $p(x)-f(x)=3 x^{2}-2 x^{3}-\sin ^{2}\left(\frac{\pi}{2} x\right)$ :

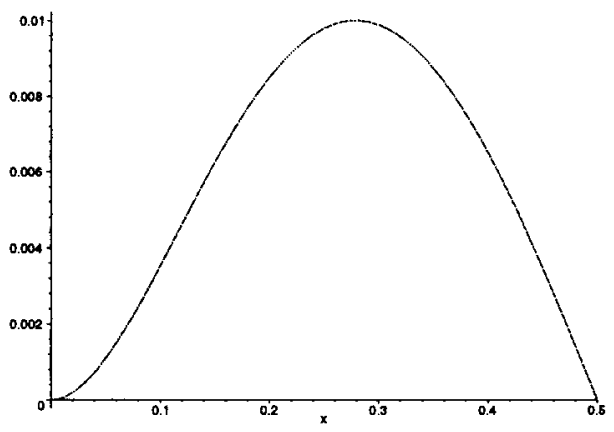

EXAMPLE 3.3. (Quasi-variable) Given any continuous function $f: X \rightarrow[0,1]$ and quasi-measure $\mu \in Q(X)$ one may show that we obtain a probability measure $\mu_{f}$ on $[0,1]$ by $\mu_{f}(A)=\mu\left(f^{-1}(A)\right)$ for all open or closed sets $A \subset[0,1]$. Let $X=D=$ unit disk, let $\mu$ be the quasi-probability in Example 3.2 and define $f: D \rightarrow[0,1]$ by $r e^{i \theta} \mapsto r$. Then $\mu_{f}[0, r]$ is the probability of at least two points being within a radius $r$ of the origin. Using the formula (2) with $D_{x}$ being the disk with radius $x$ we find that

$$
F_{X}(x)=\mu_{f}[0, x)=\sin ^{2}\left(\frac{\pi}{2} x^{2}\right), \quad x \in[0,1],
$$

where $F_{X}(x)$ is the cumulative distribution function of a random variable $X$ on $[0,1]$. Differentiating we find the Radon-Nikodym derivative of $\mu_{f}$ with respect to the Lebesgue measure on $[0,1], f_{X}(x)=2\left(\sin \frac{1}{2} \pi x^{2} \cos \frac{1}{2} \pi x^{2}\right) \pi x$ (of course $f_{X}(x)$ is the probability distribution function of $X$ ). Now we may calculate the expectation of $X: E(X)=\int_{0}^{1} x f_{X}(x) d x$. But what is $X$ ? The variable $X$ interpreted in this example is the distance of the second point from the origin.

Remark 3. Notice that the argument above relies on the fact that any continuous map $f: D \rightarrow[0,1]$ maps $\mu$ onto a probability measure in $[0,1]$. 
This enables us to calculate directly from $\mu$ without considering the (possibly) underlying three dependent variables in $D^{3}$.

\section{REFERENCES}

1. J. F. Aarnes, Quasi-states and quasi-measures, Adv. in Math. 86 (1991), 41-67

2. J. F. Aarnes, Construction of non-subadditive measures and discretization of Borel measures, Fund. Math. 147 (1995), 213-237

3. F. F. Knutsen, Topology and the construction of extreme quasi-measures, Adv. in Math. 120 (1996), 302-321.

4. G. Taraldsen, Quasi-probability A theory with incompatible stochastic variables, Preprint NO. 7/1996, Math. NTNU

NORWEGIAN UNIVERSITY OF SCIENCE AND TECHNOLOGY

DEPARTMENT OF MATHEMATICAL SCIENCES, LADE

7491 TRONDHEIM

NORWAY 